\title{
Symmetry in Scalar Field Topology
}

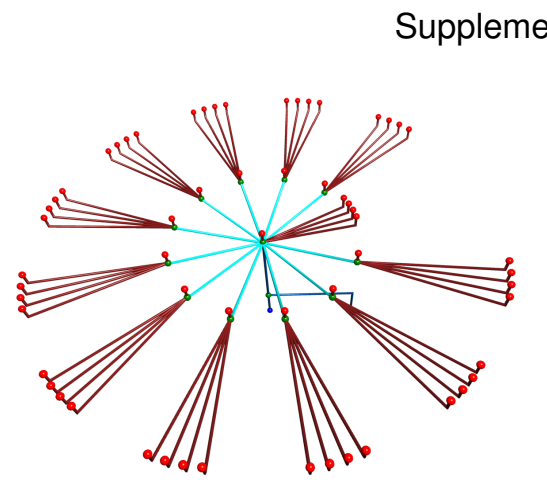

(a)

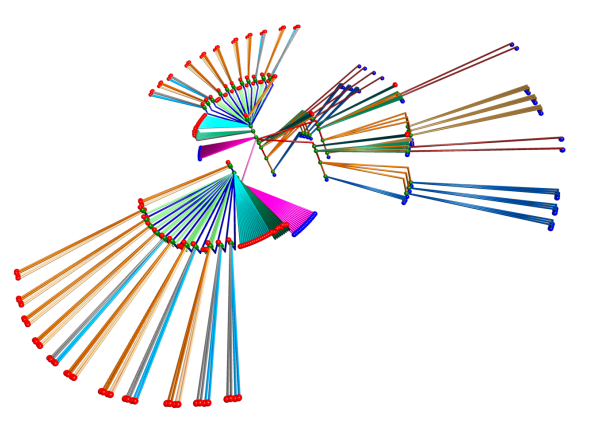

(b)

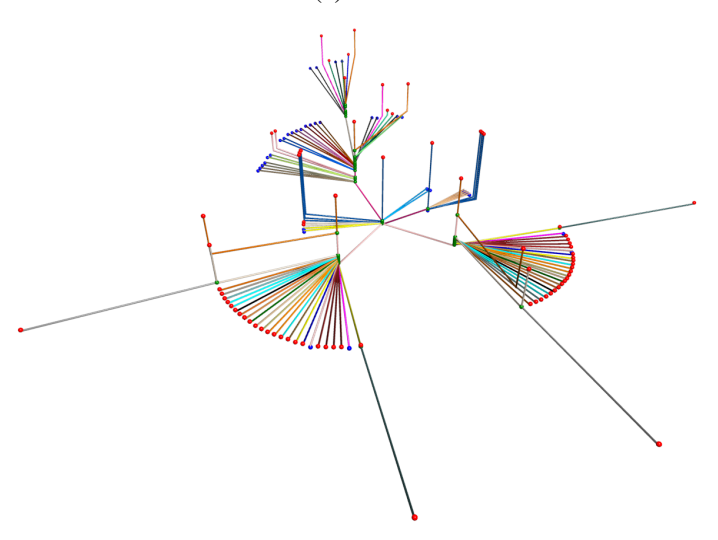

(c)

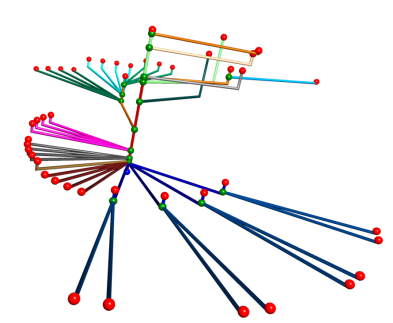

(d)

Fig. 1. Stable branch decomposition showing refined groups for (a) Buckyball, (b) Vortex simulation, (c) Neghip, and (d) Fuel.

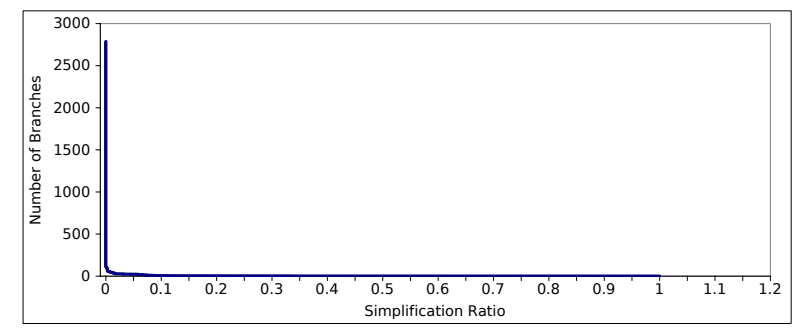

Fig. 2. Graph showing the number of branches in the contour tree plotted against simplification ratio for the Fuel data set. Initially, there is a sudden drop due to noise in the data. The value at which the graph tapers off is used as an estimate for simplification ratio and stabilisation ratio. 

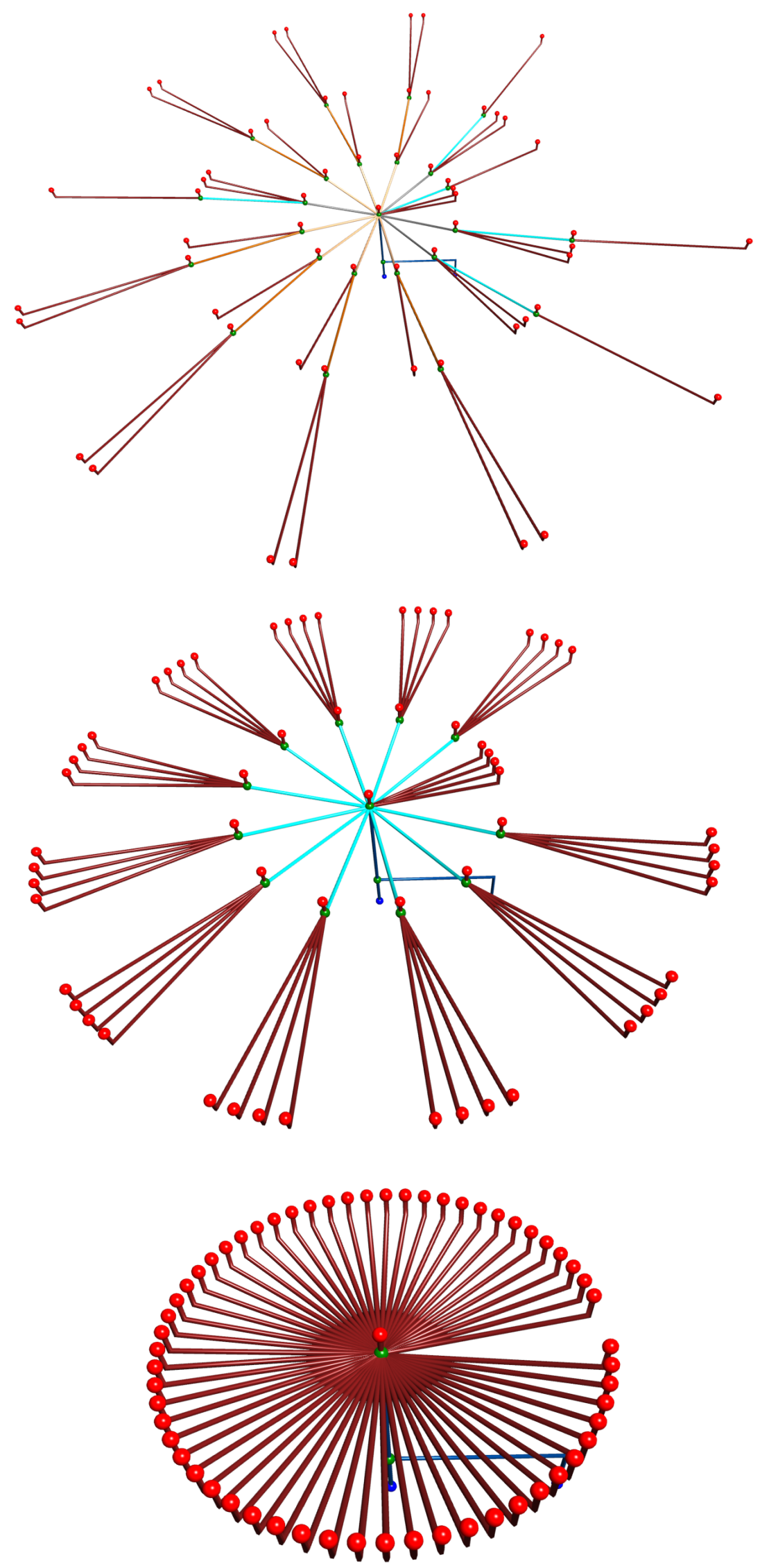

Fig. 3. Contour tree for the Buckyball data set after preprocessing using different stabilisation ratios. (a)At $1 \%$, one set of carbon atoms merge in groups of two while another set merge in groups of three. (b) At $2 \%$, carbon atoms merge in groups of five. (c) At $8 \%$, all children branches merge with the root branch and we do not see any relationship in the way the carbon atoms merge. 